\title{
Significance of TLR4, MyD88 and STAT3 expression in colorectal cancer.
}

\author{
Lei Zhou ${ }^{1 \#, ~ H u i-Z h i ~ L i a n g ~}{ }^{1 \#}$, Heng Zhang ${ }^{1}$, Xiang-Yang Wang ${ }^{2}$, Dan $\mathrm{Xu}^{1}$, Jie Wu ${ }^{\text {* }}$ \\ ${ }^{1}$ Department of Gastroenterology and Key Laboratory for Molecular Diagnosis of Hubei Province, The Central Hospital \\ of Wuhan, Tongji Medical College, Huazhong University of Science and Technology, Wuhan, Hubei, PR China
}

${ }^{2}$ Department of Gastrointestinal Surgery, The Central Hospital of Wuhan, Tongji Medical College, Huazhong University of Science and Technology, Wuhan, Hubei, PR China

\#These authors contributed equally to this work.

\begin{abstract}
Objective: The aim of this study was to investigate the expression status of toll-like receptor 4 (TLR4), myeloid differentiation primary response protein 88 (MyD88) and signal transducers and activators of transcription 3 (STAT3) in colorectal cancer (CRC) by immunohistochemistry (IHC), and explore the relationship between their expression and clinicopathological features.

Materials and methods: Expression of TLR4/MyD88/STAT3 was detected using IHC staining in 20 colorectal adenomas, $40 \mathrm{CRC}$ samples and 40 matched non-tumor tissues, and the correlations between their immunoscores and clinicopathological variables were analysed.

Results: The IHC results showed that the tumor sites exhibited significantly higher levels of all these three proteins compared to the adenoma and adjacent non-tumor sites. Further data analysis showed the expression of TLR4, MyD88 and STAT3 was positively correlated with each other in CRC tissue samples. Among clinicopathological parameters, overexpression of TLR4 was significantly correlated with lymph node metastasis (LNM) $(\mathbf{P}=\mathbf{0 . 0 2 3})$, MyD88 expression was significantly associated with the degree of differentiation $(P=0.006)$, LNM $(P=0.009)$ and Tumor-Node-Metastasis (TNM) stage $(P=0.018)$, whereas STAT3 expression was significantly associated with tumor size $(P=0.013), L N M(P=0.001)$ and TNM stage (P=0.002).

Conclusion: The overexpression of TLR4/MyD88/STAT3 proteins may play an important role in the pathogenesis of CRC.
\end{abstract}

Keywords: Colorectal cancer, TLR4, MyD88, STAT3, Immunohistochemistry.

Accepted on October 28, 2017

\section{Introduction}

Colorectal cancer (CRC) is the third most commonly diagnosed cancer in males and the second in females, with over 1.3 million new cancer cases and 693,900 deaths estimated to have occurred in 2012 [1]. Over the recent decades, the incidence of CRC has been increasing dramatically in several developing countries and has attracted wide attention [2-5]. The theory of adenoma-carcinoma sequence is now accepted as the mechanism of CRC [6]. While many of the oncogenes and tumor suppressor genes that are involved in the occurrence and progression of $\mathrm{CRC}$ have been identified, the molecular mechanisms of CRC has not been fully determined.

Toll-like receptors (TLRs) is a type I transmembrane receptor that was originally identified in Drosophila for its involvement in embryo development [7]. They act as pathogen recognition receptors and play key roles in both innate and adaptive immune responses [8]. There are 10 human isoforms of TLRs (TLR1 to 10) have been identified. Human TLRs are expressed in various cell types, including immune cells, intestinal epithelial cells and tumor cells $[9,10]$. TLR4 is the best characterized pathogen recognition receptor. There is increasing evidence suggests that TLR4 plays important roles in cancers [11]. TLR4 specifically binds to an adaptor molecule, myeloid differentiation primary response protein 88 (MyD88), resulting in the production of proinflammatory cytokines, such as IL-6 or TNF. Current studies suggest MyD88-dependent signaling has been shown to be critical for oncogenesis and tumor progression, such as lymphoma and hepatocellular carcinoma $[12,13]$.

Signal transducers and activators of transcription (STATs) are cytoplasmic transcription factors, and STATs are key mediators of cytokine and growth factor signalling pathways [14]. STAT3 is a member of the STATs family and is also a target gene of TLR4/MyD88 signaling [15,16]. In addition, prolonged activation of STAT3 plays a significant role in promoting development and progression of many cancers, such as lung cancer, hepatocellular carcinoma and breast cancer [17]. 
Currently, few studies have been published on the relations between TLR4/MyD88/STAT3 expression and clinicopathological characteristics in CRC patients. In this study, we investigated the expressions of TLR4, MyD88 and STAT3 in colorectal adenomas, CRC and peritumoral tissues by performing IHC and we evaluated the relationship between these proteins and the clinicopathologic factors.

\section{Materials and Methods}

\section{Patients and tissue specimens}

We obtained colorectal adenomas from 20 patients and tumors and peritumoral colorectal tissues from $40 \mathrm{CRC}$ patients who had undergone endoscopic resection or surgery at the Central Hospital of Wuhan in 2016. None of the 40 CRC patients had received chemotherapy or radiotherapy prior to surgery. Tissue samples were collected immediately following endoscopic resection or surgery. The non-malignant colorectal tissues were at least $2 \mathrm{~cm}$ in distance from the tumor margin. The tissue samples were fixed in $4 \%$ paraformaldehyde and embedded in paraffin for histopathological diagnosis and immunohistochemical staining. The diagnoses were confirmed by histopathological study. Detailed clinical and histopathological information was collected based on case records and pathologic reports. Table 1 shows the general clinicopathological features of these 40 patients with CRC. Tumors located proximal to the splenic flexure were considered proximal, whereas those located after the splenic flexure were distal. Tumor staging was determined by the TNM Classification ( $7^{\text {th }}$ edition) of the American Joint Committee on Cancer (AJCC) [18]. The present study was performed according to the guidelines of the Ethics and Research Committee of the Central Hospital of Wuhan and approved in accordance with the ethical standards of the World Medical Association Declaration of Helsinki.

\section{Immunohistochemical staining}

This was performed as previously described by the same author [19]. We used the primary antibodies were as following: a rabbit anti-TLR4 polyclonal antibody (Wuhan Boster Bioengineering Co., Ltd., China) at 1:100 dilution, a rabbit antiMyD88 polyclonal antibody (Wuhan Boster Bio-engineering Co., Ltd.) at 1:200 dilution, and a rabbit anti-STAT3 polyclonal antibody (Wuhan Boster Bio-engineering Co., Ltd.) at 1:200 dilution. Immunohistochemical staining was performed using the Vectastain Elite ABC kit (Vector Laboratories, Burlingame, CA, USA) according to the manufacturer's instructions. Negative controls were performed by replacing the primary antibody with phosphate-buffered saline (PBS).

Table 1. Clinicopathological characteristics of the 40 CRC patients.

\begin{tabular}{ll}
\hline Characteristics & Results \\
\hline Gender & \\
\hline Male & 24 \\
\hline
\end{tabular}

\begin{tabular}{|c|c|}
\hline Female & 16 \\
\hline \multicolumn{2}{|l|}{ Age (years) } \\
\hline$<65$ & 25 \\
\hline$\geq 65$ & 15 \\
\hline \multicolumn{2}{|l|}{ Tumor site } \\
\hline Distal & 25 \\
\hline Proximal & 15 \\
\hline \multicolumn{2}{|l|}{ Tumor diameter (cm) } \\
\hline$<5$ & 22 \\
\hline$\geq 5$ & 18 \\
\hline \multicolumn{2}{|l|}{ Pathological grade } \\
\hline Well differentiated & 9 \\
\hline Well-moderately differentiated & 7 \\
\hline Moderately differentiated & 15 \\
\hline Moderately-poorly differentiated & 6 \\
\hline Poorly differentiated & 3 \\
\hline \multicolumn{2}{|l|}{ Depth of invasion } \\
\hline $\mathrm{T} 1+\mathrm{T} 2$ & 5 \\
\hline $\mathrm{T} 3+\mathrm{T} 4$ & 35 \\
\hline \multicolumn{2}{|l|}{ LNM } \\
\hline No & 17 \\
\hline N1 & 11 \\
\hline N2 & 12 \\
\hline \multicolumn{2}{|l|}{ Distant metastasis } \\
\hline M0 & 34 \\
\hline M1 & 6 \\
\hline \multicolumn{2}{|l|}{ TNM stage } \\
\hline $1+11$ & 15 \\
\hline$I I I+I V$ & 25 \\
\hline \multicolumn{2}{|l|}{ Lymphovascular invasion } \\
\hline Present & 29 \\
\hline Absent & 11 \\
\hline
\end{tabular}

LNM: Lymph Node Metastasis; TNM: Tumor Node Metastasis

\section{Evaluation of immunohistochemical findings}

Each slide was evaluated independently by two observers who were blinded to clinical data. The immunostaining score of TLR4, MyD88, and STAT3 proteins was assessed by the percentage of positively stained tumor cells as described earlier: 0 , no positives staining; $1,1-25 \%$ cells positive; 2 , $26-50 \%$ cells positive; $3,51-75 \%$ cells positive; $4,76-100 \%$ cells positive $[19,20]$. Specimens with scores $\geq 2$ were labeled 
as 'positive $(+)$ '. The staining areas were imaged with a BX51 model light microscope (at x200 and x400 magnification; OLYMPUS Corporation, Tokyo, Japan).
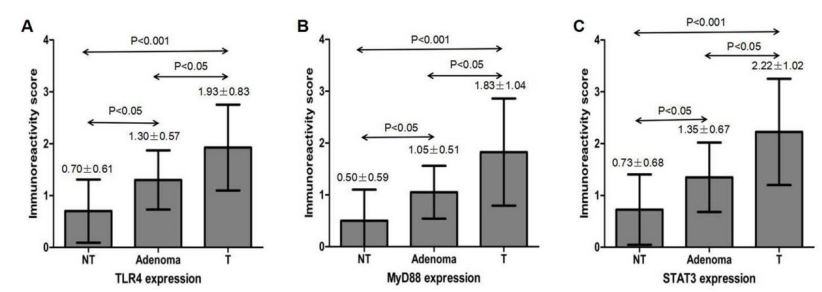

Figure 1. Comparison of TLR4, MyD88 and STAT3 expression levels in colorectal tissues. The immunostaining scores of TLR4 (A), MyD88 (B) and STAT3 $(C)$ in $T$ were significantly higher than in adenoma and NT. In addition, the immunostaining scores of TLR4 (A), MyD88 $(B)$ and STAT3 $(C)$ were significantly higher in adenoma compared to NT. Data are presented as mean \pm standard deviation; $T=C R C$ tissues; NT=adjacent non-tumor tissues.

\section{Statistical analysis}

Data were presented in terms of mean \pm standard deviation, percentage and the number of cases. The analyses that compared the expressions of TLR4, MyD88 and STAT3 between adenoma, tumor and peritumoral tissues were performed using Kruskal-Wallis test. The relationships between the levels of three proteins expression and the various clinicopathological parameters in CRC were determined by Fisher's exact test. Correlations were calculated using the Spearman's rank correlation coefficient. P-value $<0.05$ was considered to indicate a statistically significant difference in all tests. All statistical analyses were performed using SPSS version 17.0 (SPSS, Inc., Chicago, IL, USA) and GraphPad Prism version 5.0 (GraphPad Software Inc., San Diego, CA, USA).

\section{Results}

\section{The expressions of TLR4, MyDS8 and STAT3 in colorectal adenoma, $C R C$ and peritumoral colorectal tissues}

TLR4 protein expression was located in the cytoplasm and membrane, and MyD88 and STAT3 protein expression was located in the cytoplasm, as brownish-yellow granules. The expression of TLR4, MyD88 and STAT3 was seen in $25 \%$ $(5 / 20), 15 \%(3 / 20)$ and $45 \%(9 / 20)$ of the colorectal adenoma, respectively. The immunostaining score of TLR4, MyD88 and STAT3 was $1.30 \pm 0.57,1.05 \pm 0.51$ and $1.35 \pm 0.67$ in adenoma, respectively (Figure 1). Examples of adenoma tissue specimens of TLR4, MyD88 and STAT3 staining intensity are shown at (Figure 2). The expression of TLR4, MyD88 and STAT3 was seen in 77.5\% (31/40), 60\% (24/40) and 75\% (30/40) of the CRC, respectively. The immunostaining score of TLR4, MyD88 and STAT3 was $1.93 \pm 0.83,1.83 \pm 1.04$ and $2.22 \pm 1.02$ in CRC, respectively (Figure 1). Examples of CRC tissue specimens of TLR4, MyD88 and STAT3 staining intensity are shown at (Figure 3).

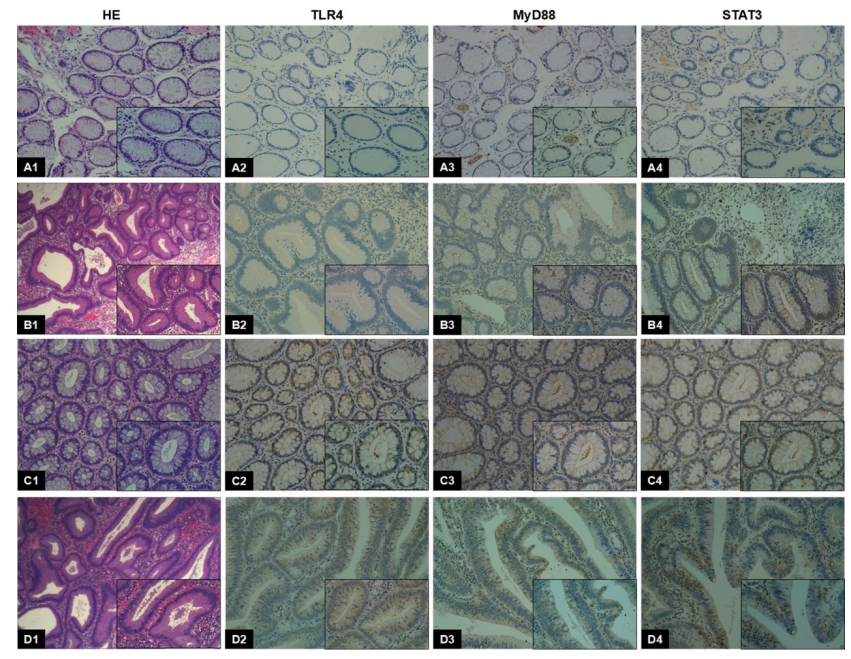

Figure 2. Detection of TLR4, MyD88 and STAT3 immunoreactivity in colorectal adenomas. (A) TLR4 (A2), MyD88 (A3) and STAT3 (A4) showed no immunoreactions in adenoma (A1). (B) No TLR4 (B2) and $M y D 88$ (B3) immunostaining were present in adenoma (B1), but STAT3 (B4) had a positive staining (immunoreactivity score=1). (C) TLR4 (C2), MyD88 (C3) and STAT3 (C4) showed positive immunoreactions in adenoma (C1), the score was 2, 1 and 2, respectively. (D) TLR4 (D2), MyD88 (D3) and STAT3 (D4) showed positive immunoreactions in adenoma (D1), the score was 3, 2 and 1, respectively. Original magnification $200 X$ and $400 X$.

The expression of TLR4, MyD88 and STAT3 was seen in $7.5 \%$ $(3 / 40), 5 \%(2 / 40)$ and $12.5 \%(5 / 40)$ of the peritumoral colorectal tissues, respectively. The immunostaining score of TLR4, MyD88 and STAT3 was $0.70 \pm 0.61,0.50 \pm 0.59$ and $0.73 \pm 0.68$ in peritumoral tissues, respectively (Figure 1). Examples of peritumoral tissue specimens of TLR4, MyD88 and STAT3 staining intensity are shown at (Figure 3). The staining intensity of TLR4, MyD88 and STAT3 was significantly higher in the CRC as compared with that of the adenoma and adjacent non-tumor tissues respectively $(\mathrm{P}<0.05$ and $\mathrm{P}<0.001$, respectively) (Figure 1 ). The staining intensity of TLR4, MyD88 and STAT3 also tended to be significantly higher in the adenoma when compared with the adjacent nontumor tissues respectively $(\mathrm{P}<0.05)$ (Figure 1).

Table 2. Correlation among TLR4, MyD88 and STAT3 in CRC tissues

\begin{tabular}{lllll}
\hline & & TLR4 & MyD88 & STAT3 \\
\hline \multirow{2}{*}{ TLR4 } & Spearman Correlation & - & 0.328 & 0.346 \\
\cline { 2 - 5 } & P-value & - & 0.039 & 0.029 \\
\hline \multirow{2}{*}{ MyD88 } & Spearman Correlation & - & - & 0.494 \\
\cline { 2 - 5 } & P-value & - & - & 0.001 \\
\hline
\end{tabular}

\section{Correlation among TLR4, MyDS8 and STAT3 expression in $C R C$}

In CRC, TLR4 and MyD88 protein expression was significantly positively correlated $(\mathrm{r}=0.328, \mathrm{P}=0.039)$ as was MyD88 and STAT3 protein expression $(r=0.494, \mathrm{P}=0.001)$ 
(Table 2). TLR4 and STAT3 protein expression was positively correlated $(\mathrm{r}=0.346, \mathrm{P}=0.029)$ (Table 2$)$.

Table 3. Relationship between clinicopathological features and expression of TLR4, MyD88 and STAT3 in CRC tissues.

\begin{tabular}{|c|c|c|c|c|c|c|c|}
\hline \multirow[b]{2}{*}{ Variables } & \multirow[b]{2}{*}{$\mathrm{n}$} & \multicolumn{2}{|c|}{ TLR4 (+) } & \multicolumn{2}{|c|}{ MyD88 (+) } & \multicolumn{2}{|c|}{ STAT3 (+) } \\
\hline & & No. (\%) & $\mathrm{P}$ & No. (\%) & $P$ & No. $(\%)$ & $P$ \\
\hline \multicolumn{8}{|l|}{ Gender } \\
\hline Male & 24 & $20(83.3)$ & 0.441 & $12(50.0)$ & 0.188 & $19(79.2)$ & 0.482 \\
\hline Female & 16 & $11(68.8)$ & & $12(75.0)$ & & $11(68.8)$ & \\
\hline
\end{tabular}

\begin{tabular}{|c|c|c|c|c|c|c|c|}
\hline \multicolumn{8}{|c|}{ Age (years) } \\
\hline$<65$ & 25 & $19(76.0)$ & 1 & $14(56.0)$ & 0.739 & $17(68.0)$ & 0.268 \\
\hline$\geq 65$ & 15 & $12(80.0)$ & & $10(66.7)$ & & $13(86.6)$ & \\
\hline \multicolumn{8}{|c|}{ Tumor site } \\
\hline Distal & 25 & $18(72.0)$ & 0.44 & $16(64.0)$ & 0.526 & $18(72.0)$ & 0.714 \\
\hline Proximal & 15 & $13(86.7)$ & & $8(53.3)$ & & $12(80.0)$ & \\
\hline \multicolumn{8}{|c|}{ Tumor size $(\mathrm{cm})$} \\
\hline$<5$ & 22 & $17(77.2)$ & 1 & $13(59.1)$ & 1 & $13(59.1)$ & 0.013 \\
\hline$\geq 5$ & 18 & $14(77.7)$ & & $11(61.1)$ & & $17(94.4)$ & \\
\hline
\end{tabular}

\begin{tabular}{|c|c|c|c|c|c|c|c|}
\hline \multicolumn{8}{|c|}{ Pathological grade* } \\
\hline Group I & 31 & $22(70.9)$ & 0.089 & $15(48.4)$ & 0.006 & $21(67.7)$ & 0.081 \\
\hline Group II & 9 & $9(100.0)$ & & $9(100.0)$ & & $9(100.0)$ & \\
\hline \multicolumn{8}{|l|}{ LNM } \\
\hline Negative & 17 & $10(58.8)$ & 0.023 & $6(35.3)$ & 0.009 & $8(47.1)$ & 0.001 \\
\hline Positive & 23 & 21 (91.3) & & $18(78.3)$ & & $22(95.6)$ & \\
\hline
\end{tabular}

\section{TNM stage}

\begin{tabular}{|c|c|c|c|c|c|c|c|}
\hline $1+\|$ & 15 & $9(60.0)$ & 0.057 & $5(33.3)$ & 0.018 & $7(46.7)$ & 0.002 \\
\hline III+IV & 25 & $22(88.0)$ & & $19(76.0)$ & & $23(92.0)$ & \\
\hline \multicolumn{8}{|c|}{ Lymphovascular invasion } \\
\hline Absent & 11 & $10(90.9)$ & 0.399 & $6(54.5)$ & 0.727 & $7(63.6)$ & 0.417 \\
\hline Present & 29 & $21(72.4)$ & & $18(62.1)$ & & $23(79.3)$ & \\
\hline
\end{tabular}

${ }^{*} \mathrm{CRC}$ was subdivided into two groups according to differentiation degree; group I was defined as well, well-moderately and moderately differentiated and group II was defined as moderately-poorly and poorly differentiated. LNM: Lymph Node Metastasis; TNM: Tumor Node Metastasis

\section{Relationship between clinicopathological features and expression of TLR4, MYDS8 and STAT3 in CRC}

When analyzing the relationship between protein expression and clinicopathological features, we found that the expression of TLR4 was significantly correlated with LNM $(\mathrm{P}=0.023)$ and potentially related to the pathological grade $(\mathrm{P}=0.089)$ and TNM stage $(\mathrm{P}=0.057)$ (Table 3$)$. Expression of MyD88 was significantly associated with cancer histology $(\mathrm{P}=0.006)$, LNM $(\mathrm{P}=0.009)$ and TNM stage $(\mathrm{P}=0.018)$ (Table 3$)$. Expression of
STAT3 was significantly associated with tumor size $(\mathrm{P}=0.013)$, LNM $(\mathrm{P}=0.001)$ and TNM stage $(\mathrm{P}=0.002)$ and potentially related to histologic type $(\mathrm{P}=0.081)$ (Table 3$)$. The expression of each protein was not significantly different according to gender, age, tumor site and lymphovascular invasion.

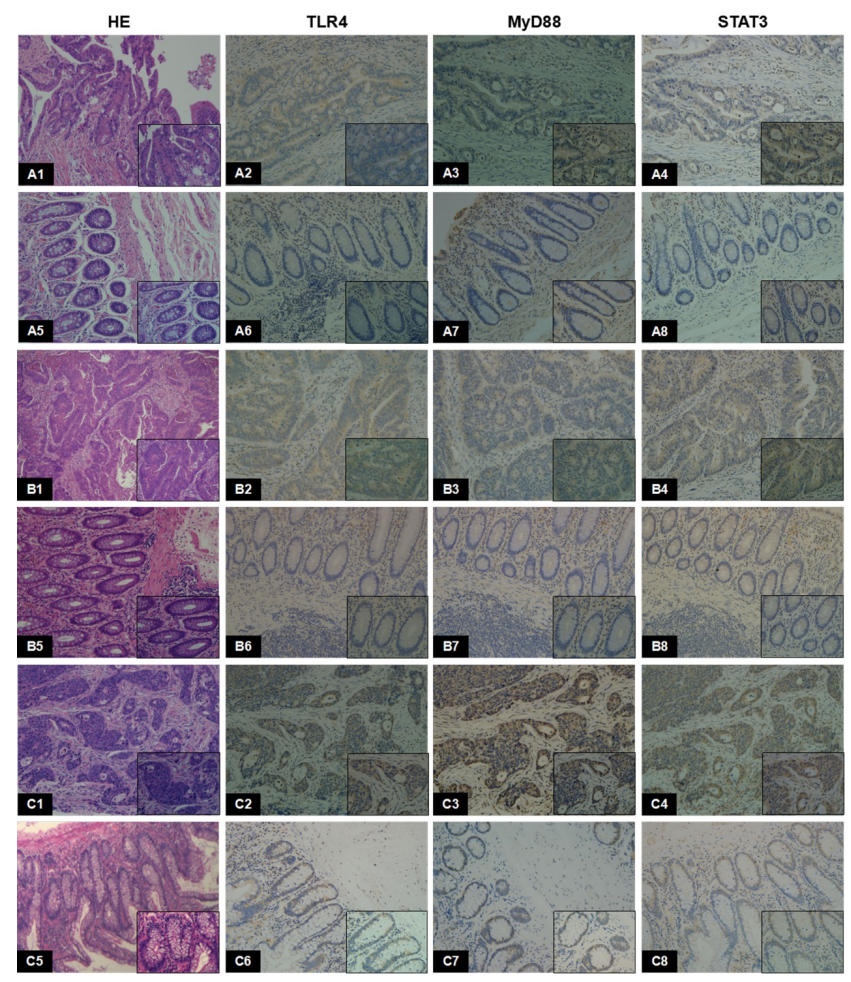

Figure 3. Detection of TLR4, MyD88 and STAT3 immunoreactivity in CRC and adjacent non-tumor tissues. (A) TLR4, MyD88 and STAT3 showed positive immunoreactions in well-moderately differentiated $C R C$ (A1) (immunoreactivity score $=2,1$ and 2, respectively), but no immunoreactions in matched non-tumor tissues (A5). (B) TLR4, MyD88 and STAT3 showed positive immunoreactions in moderately differentiated CRC (B1) (immunoreactivity score =4, 2 and 3, respectively), but no immunoreactions in matched non-tumor tissues (B5). (C) TLR4, MyD88 and STAT3 showed positive immunoreactions in poorly differentiated $C R C$ (C1) (immunoreactivity score $=3,3$ and 4, respectively) and matched non-tumor tissues (C5) (immunoreactivity score $=2,2$ and 2, respectively). Original magnification $200 X$ and $400 X$.

\section{Discussion}

In recent years, there is increasing evidence that TLR4 signaling pathway is involved in tumorigenesis [11]. MyD88 plays a critical role in TLR signaling. Some experimental studies have identified the contribution of TLR4/MyD88 signaling to tumor development and progression [21-23]. In this study, we observed that TLR4 and MyD88 signaling factors were highly expressed in CRC compared with adjacent non-tumor tissues and adenoma, and this result was near to Wang et al. who found that TLR4 and MyD88 immunostaining was absent or very weak in normal mucosa and adenoma, but a higher proportion of CRC cases showed positive expression of TLR4 and MyD88 [21]. Similar result had been reported in breast cancer by Chen et al. [22]. A correlation between TLR4 and MyD88 expression was detected. This agreed with Wang 
et al and also agreed with Kim et al. who found that there was a positive correlation between the expression of TLR4 and MyD88 in CRC and ovarian epithelial cancer (OEC) through IHC, respectively [21,23]. Collectively, our study provided us with preliminary results on the possible involvement of TLR4/ MyD88 signaling pathway in pathogenesis of CRC.

In the present study, there was a statistically significant association between TLR4 positivity and LNM. This result agreed with Ehsan et al. who found that the incidence of LNM was higher in patients with high TLR4 expression in the breast tumor cells [24]. Moreover, we noted that the expression level of TLR4 tended to be higher in poorly differentiated and advanced tumors, and this result agreed with Wang et al. who found that TLR4 expression was significantly associated with TNM stage in CRC [21]. Similar result had been reported in breast cancer by Chen et al. [22]. These findings indicated that increased TLR4 expression in CRC might be associated with tumor progression.

The current study showed that MyD88 positivity was significantly associated with LNM and TNM stage. This agreed with Chen et al, who found that the expression of MyD88 was significantly associated with axillary LNM and tumor staging in breast cancer [22]. Moreover, in the present study there was a statistically significant association between high expression of MyD88 and histological grade. This result was similar to Wang et al. who found that MyD88 expression had a significant association with differentiation grade in CRC [21]. Similar result had been reported by Kim et al. who found that there was a significant correlation between MyD88 expression and tumor stage and MyD88 was an independent prognostic predictor in OEC [23]. Our findings suggested that MyD88 may partially contribute to the progression of CRC.

STAT3, as an important downstream effector of the TLR4/ MyD88 signaling has been identified $[15,16]$. Recently, many studies have demonstrated that the constitutively activated STAT3 protein contributes to malignant transformation and tumor progression [17]. In the present study, the expression of STAT3 was found to be more intense in CRC compared with adjacent non-tumor tissues and adenoma, and STAT3 protein expression was significantly associated with tumor size and potentially related to histologic type. These results were similar to the observation by Lee et al. conducted on 20 cases of colorectal adenomas and 39 cases of colorectal adenocarcinomas [20]. While they found the expression of STAT3 was increased in the adenocarcinomas as compared with the adenomas and was significantly higher in the tumors with a larger size. Artas et al. found that the expression of STAT3 was significantly higher in colon adenocarcinoma than in normal colon mucosa, and was significantly related to the tumor differentiation [25]. In addition, the current study showed that the STAT3 protein expression was significantly related to LNM and TNM stage. Jin et al. found that the 3- and 5-year survival rates of STAT3 protein-positive CRC patients were lower than the rates in STAT3 protein-negative patients [26]. Our findings might add evidence that STAT3 was participated with the development and progression of CRC.
Our results showed that the expressions of TLR4, MyD88 and STAT3 were increased in CRC as compared with the expressions in colorectal adenoma and adjacent normal mucosa, and the staining intensity was increased with the progression from adenoma to CRC. The results suggested that these proteins may play a driving role towards colorectal carcinogenesis with the progression from adenoma to carcinoma. Especially, our study found that the expression of TLR4, MyD88 and STAT3 was positively correlated with each other in CRC tissues. Further data analysis showed that all these three proteins had correlation with lymph node metastasis in CRC. Evidence from the literature and our findings indicated that the expression of TLR4, MyD88 and STAT3 may be interdependent, and that each has their own role and prognostic value in $\mathrm{CRC}$; elevated these three proteins expression may partly contribute to the development and progression of CRC.

The limitations that we faced in the current study were the small number of CRC cases as they were 40 cases only, also the association between TLR4/MyD88/STAT3 signaling and cancer mortality has not been investigated in clinical samples.

\section{Conclusion}

Our study revealed that TLR4, MyD88 and STAT3 were overexpressed in the tumor cells of CRC using IHC and these proteins expression was significantly associated with lymph node metastasis. It is now more evident that TLR4/MyD88/ STAT3 proteins may be one of the major signaling factors involved in the pathogenesis of CRC. However, further investigation is required to identify the detailed mechanisms of these proteins in colorectal carcinogenesis.

\section{Acknowledgment}

This study was funded by a grant from the Research Funding for Health and Family Planning Commission of Wuhan Municipality (grant no. WX16D38).

\section{References}

1. International Agency for Research on Cancer. GLOBOCAN 2012: Estimated Cancer Incidence, Mortality and Prevalence Worldwide in 2012. World Health Organization.

2. Yazdizadeh B, Jarrahi AM, Mortazavi H, Mohagheghi MA, Tahmasebi S. Time trends in the occurrence of major GI cancers in Iran. Asian Pac J Cancer Prev 2005; 6: 130-134.

3. Khuhaprema T, Srivatanakul P. Colon and rectum cancer in Thailand: an overview. Jpn J Clin Oncol 2008; 38: 237-243.

4. Ibrahim KO, Anjorin AS, Afolayan AE, Badmos KB. Morphology of colorectal carcinoma among Nigerians: a 30-year review. Niger J Clin Pract 2011; 14: 432-435.

5. Dai Z, Zheng RS, Zou XN, Zhang SW, Zeng HM, Li N, Chen WQ. Analysis and prediction of colorectal cancer incidence trend in China. Zhonghua Yu Fang Yi Xue Za Zhi 2012; 46: 598-603. 
6. Leslie A, Carey FA, Pratt NR, Steele RJ. The colorectal adenoma-carcinoma sequence. Br J Surg 2002; 89: 845-860.

7. Hashimoto C, Hudson KL, Anderson KV. The Toll gene of Drosophila, required for dorsal-ventral embryonic polarity, appears to encode a transmembrane protein. Cell 1988; 52: 269-279.

8. Chen K, Huang J, Gong W, Iribarren P, Dunlop NM, Wang JM. Toll-like receptors in inflammation, infection and cancer. Int Immunopharmacol 2007; 7: 1271-1285.

9. Frosali S, Pagliari D, Gambassi G, Landolfi R, Pandolfi F, Cianci R. How the Intricate Interaction among Toll-Like Receptors, Microbiota, and Intestinal Immunity Can Influence Gastrointestinal Pathology. J Immunol Res 2015; 2015: 489821

10. Yeh DW, Huang LR, Chen YW, Huang CF, Chuang TH. Interplay between inflammation and stemness in cancer cells: The role of toll-like receptor signaling. J Immunol Res 2016; 2016: 4368101.

11. Mai CW, Kang YB, Pichika MR. Should a Toll-like receptor 4 (TLR-4) agonist or antagonist be designed to treat cancer? TLR-4: its expression and effects in the ten most common cancers. Onco Targets Ther 2013; 6: 1573-1587.

12. Ngo VN, Young RM, Schmitz R, Jhavar S, Xiao W, Lim KH, Kohlhammer H, Xu W, Yang Y, Zhao H, Shaffer AL, Romesser P, Wright G, Powell J, Rosenwald A, MullerHermelink HK, Ott G, Gascoyne RD, Connors JM, Rimsza LM, Campo E, Jaffe ES, Delabie J, Smeland EB, Fisher RI, Braziel RM, Tubbs RR, Cook JR, Weisenburger DD, Chan WC, Staudt LM. Oncogenically active MYD88 mutations in human lymphoma. Nature 2011; 470: 115-119.

13. Jia RJ, Cao L, Zhang L, Jing W, Chen R, Zhu MH, Guo SW, Wu GB, Fan XY, Wang H, Zhang YY, Zhou XY, Zhao J, Guo YJ. Enhanced myeloid differentiation factor 88 promotes tumor metastasis via induction of epithelialmesenchymal transition in human hepatocellular carcinoma. Cell Death Dis 2014; 5: e1103.

14. Darnell JE Jr. STATs and gene regulation. Science 1997; 277: 1630-1635.

15. Yamawaki Y, Kimura H, Hosoi T, Ozawa K. MyD88 plays a key role in LPS-induced Stat3 activation in the hypothalamus. Am J Physiol Regul Integr Comp Physiol 2010; 298: R403-410.

16. Liu BS, Stoop JN, Huizinga TW, Toes RE. IL-21 enhances the activity of the TLR-MyD88-STAT3 pathway but not the classical TLR-MyD88-NF- $\kappa$ B pathway in human B cells to boost antibody production. J Immunol 2013; 191: 4086-4094.
17. Yuan J, Zhang F, Niu R. Multiple regulation pathways and pivotal biological functions of STAT3 in cancer. Sci Rep 2015; 5: 17663 .

18. Edge SB, Byrd DR, Compton CC, Fritz AG, Greene FL, Trotti A. AJCC cancer staging manual (7th Edn). SpringerVerlag, New York, 2010.

19. Zhou L, Wang Y, Tian DA, Yang J, Yang YZ. Decreased levels of nitric oxide production and nitric oxide synthase-2 expression are associated with the development and metastasis of hepatocellular carcinoma. Mol Med Rep 2012; 6: 1261-1266.

20. Lee SW, Ahn YY, Kim YS, Kang SB, Nam SW, Lee DS, Jeong HY, Kim JM. The Immunohistochemical Expression of STAT3, Bcl-xL, and MMP-2 Proteins in Colon Adenoma and Adenocarcinoma. Gut Liver 2012; 6: 45-51.

21. Wang EL, Qian ZR, Nakasono M, Tanahashi T, Yoshimoto K, Bando Y, Kudo E, Shimada M, Sano T. High expression of toll-like receptor 4/myeloid differentiation factor 88 signals correlates with poor prognosis in colorectal cancer. Br J Cancer 2010; 102: 908-915.

22. Chen X, Zhao F, Zhang H, Zhu Y, Wu K. Significance of TLR4/MyD88 expression in breast cancer. Int $\mathrm{J}$ Clin Exp Pathol 2015; 8: 7034-7039.

23. Kim KH, Jo MS, Suh DS, Yoon MS, Shin DH, Lee JH, Choi KU. Expression and significance of the TLR4/MyD88 signaling pathway in ovarian epithelial cancers. World J Surg Oncol 2012; 10: 193.

24. Ehsan N, Murad S, Ashiq T, Mansoor MU, Gul S, Khalid S, Younas M. Significant correlation of TLR4 expression with the clinicopathological features of invasive ductal carcinoma of the breast. Tumour Biol 2013; 34: 1053-1059.

25. Artaş G, Ozercan HI. The expression of STAT3, BCL-XL and MMP-2 proteins in colon adenocarcinomas and their relationship with prognostic factors. Turk Patoloji Derg 2014; 30: 178-183.

26. Jin C, Wang A, Chen J, Liu X, Wang G. Relationship between expression and prognostic ability of PTEN, STAT3 and VEGF-C in colorectal cancer. Exp Ther Med 2012; 4: 633-639.

\section{*Correspondence to}

Jie Wu

Department of Gastroenterology and Key Laboratory for Molecular Diagnosis of Hubei Province

The Central Hospital of Wuhan

PR China 